\title{
Assessment of a chemiluminescent universal probe for taxonomical and epidemiological investigations of Aeromonas sp isolates
}

\author{
P E Carey, A Eley, M H Wilcox
}

\begin{abstract}
Aims-To assess a chemiluminescent universal probe for taxonomical and epidemiological investigations of Aeromonas sp isolates.

Methods-Total DNA was extracted from 69 well characterised Aeromonas sp strains and digested with the restriction endonucleases Sma I or Pst I. Following electrophoresis, the resulting fragments were transferred to a nylon membrane where they were hybridised to a commercially available universal probe of $16 S+$ $23 S$ rRNA. The banding patterns (ribotypes) were made visible by enhanced chemiluminescence.

Results-Both restriction endonucleases produced heterogeneous ribotypes so that no allocation could be made to any of the control genospecies tested. For $A$ hydrophila and $A$ caviae, however, groups of strains (mostly from the same patient) could be identified by indistinguishable banding patterns. A relatively high proportion (36\%) of $A$ sobria strains were non-typable.
\end{abstract}

Conclusions-Although this universal chemiluminescent probe is user friendly, it is unsuitable for taxonomical investigations of Aeromonas sp. It is useful in epidemiological studies of $A$ hydrophila and $A$ caviae, but is of less value for $A$ sobria.

$(\mathcal{O}$ Clin Pathol 1994;47:642-646)

Although at least 11 phenospecies (distinguishable on the basis of a limited number of biochemical characteristics) of Aeromonas sp are now recognised, ${ }^{1}$ it is only very recently that essentially biochemical identification schemes have been described for identifying clinical Aeromonas isolates. ${ }^{23}$ However, several phenospecies consist of a number of genetically distinct groups (genospecies) that are biochemically similar. Hence at the DNA level, the phenospecies have been assigned to at least 14 hybridisation groups or genospecies. Studies of hybridisation groups to determine DNA relatedness use methods that are usually confined to reference laboratories as they are technically demanding, labour intensive, and involve radioisotopes. Similar criticisms have been made of $16 \mathrm{~S}$ rRNA sequencing, even though it is the most precise method to date for determining bacterial phylogeny. ${ }^{4} \mathrm{~A}$ different approach investigating the determination of rRNA gene restriction fragment length polymorphisms (RFLPs), also called ribotyping, has been described for use in taxonomy. ${ }^{5}$ In two separate studies of Aeromonas strains it was concluded that there was no obvious correlation between taxonomic groups and RFLPs, and that the association between RFLP patterns and hybridisation groups required further investigation. ${ }^{67}$

We have recently examined Aeromonas spp isolated from children with gastroenteritis using three phenotypic schemes, each based on biochemical reactions, ${ }^{8}$ and also by whole cell and outer membrane protein (OMP) profiling. ${ }^{9}$ Although whole cell protein profiles were unhelpful for speciation, OMP patterns were similar for $83 \%$ and $63 \%$ of $A$ caviae and $A$ hydrophila strains, respectively; extremely heterogeneous OMP patterns were observed with $A$ sobria strains.

\section{Methods}

A total of 69 Aeromonas strains was studied, including 17 and 39 faecal isolates from adults and children, respectively, who were investigated for gastroenteritis in Sheffield. Ten of the 13 reference strains (HG2, HG3, HG5A, HG5B, HG6, HG8, HG9, HG10, HG11, HG12) were kindly supplied by $M$ Altwegg (Institute of Medical Microbiology, University of Zurich, Switzerland) and have been described elsewhere. ${ }^{1}$ The remaining three organisms were American Type Culture Collection strains ATCC 15468, 7966, and 43979 ( $A$ caviae (HG4), $A$ hydrophila (HG1), and $A$ sobria (HG7), respectively). All clinical isolates were oxidase positive, fermentative, Gram negative bacilli, resistant to both low $(10 \mu \mathrm{g} \mathrm{disc})$ and high (150 $\mu \mathrm{g}$ disc) concentrations of the vibriostatic agent 0129 . These isolates have been speciated using conventional biochemical methods, the suicide phenomenon, API $20 \mathrm{NE}$ and haemolysin production, ${ }^{8}$ and by whole cell and OMP analysis using sodium dodecyl sulphate polyacrylamide gel electrophoresis (SDS-PAGE)'; phenospecies designations were either $A$ hydrophila, $A$ sobria, or $A$ caviae.

All strains were examined blind in triplicate and were decoded after ribotyping. Ribotyping was performed, as described before, ${ }^{10}$ but with modifications. Overnight growth from one plate of blood agar was removed using a sterile swab and total DNA extracted." After measuring the amount of DNA by fluorometry (Hoefer) $1 \mu \mathrm{g}$ DNA was digested with the restriction endonucleases 
Sma I or Pst I (Gibco BRL), as recommended by the manufacturer. Resulting fragments were separated by electrophoresis through agarose gels in TRIS-borate-EDTA buffer and stained with ethidium bromide $(0.5$ $\mu \mathrm{g} / \mathrm{ml}$ ) for 30 minutes. DNA bands were visualised using an ultraviolet transilluminator and the fragments transferred to a nylon membrane (Hybond N, Amersham) using the method of Southern, as described by Maniatis et al. ${ }^{12} \mathrm{~A}$ commercially available universal probe of $16 \mathrm{~S}+23 \mathrm{~S}$ rRNA (Boehringer Mannheim) was used to hybridise the DNA fragments resulting from the digestion of total DNA with restriction endonuclease. Measurement of the fragment size was achieved by comparison with a DNA marker ( $\lambda$ DNA Hind III/EcoR I digest) containing fragments of known size. The DNA marker was run on the same gel as the samples, and the same DNA was used as a probe for the marker. After boiling to produce single strands this DNA was labelled in the same way as the rRNA probe, and both probes were then added to the same hybridisation buffer. Both probes were labelled using the Enhanced Chemiluminescent (ECL) Direct Nucleic Acid Labelling and Detection Kit (Amersham UK). The nylon filters were placed in hybridisation buffer (Amersham) for one hour at $42^{\circ} \mathrm{C}$. This is a specially optimised buffer which protects the peroxidase labelling reagent during this step. Although the formulation of the buffer is not publicly known, it does contain $6 \mathrm{M}$ urea and a novel rate enchancer to increase sensitivity. Before use, $0.5 \mathrm{M} \mathrm{NaCl}$ and $5 \% \mathrm{w} / \mathrm{v}$ blocking reagent were added to the buffer and dissolved by heating to $42^{\circ} \mathrm{C}$ for one hour. For hybridisation, the labelled probes were added to the hybridisation buffer and incubated overnight at $42^{\circ} \mathrm{C}$ with agitation in a shaking water bath After hybridisation filters were washed twice in primary wash buffer (6M urea, $0.4 \%$ SDS,
$0.5 \times$ SSC where $20 \times \mathrm{SSC}=0.3 \mathrm{M}$ sodium citrate, $3 \mathrm{M}$ sodium chloride, $\mathrm{pH} 7 \cdot 0$ ) with agitation for 20 minutes each at $42^{\circ} \mathrm{C}$. This was followed by two further washes in secondary wash buffer $(2 \times \mathrm{SSC})$ for five minutes each at room temperature.

Detection reagents (Amersham) were mixed and added to the membrane under darkroom conditions, and the banding pattern was recorded by exposure to autoradiography film (Hyperfilm-ECL, Amersham) for one hour.

\section{Results}

rRNA GENE RESTRICTION PATTERNS USING

Sma I

The universal probe $(16 S+23 S$ rRNA) hybridised to fragments of Sma I digested total genomic DNA of the reference strains (fig 1). Although the intensity of some of the hybridisation bands was much stronger than others, which made comparison difficult, between three and 10 bands were seen and all hybridisation groups produced different patterns. Both high (5 to 21 kilobases) and low molecular weight bands (below $1 \cdot 3$ to 4.9 kilobases) were observed, although those above 5 kilobases were usually more easily visible.

As with the reference strains, the three phenospecies groups of clinical isolates all had heterogeneous band patterns (predominantly over 5 kilobases), making allocation to a phenospecies on the basis of ribotype pattern alone impossible. However, six pairs of $A$ caviae strains were indistinguishable and after decoding it was established that each pair of strains was from the same patient. A collection of four $A$ caviae strains ( $\mathrm{SCH}$ $35 / 36 / 37 / 38$ ) (fig 2) was also shown to be indistinguishable and comprised two pairs of strains from different patients on the same ward. Furthermore, four pairs of $A$ hydrophila
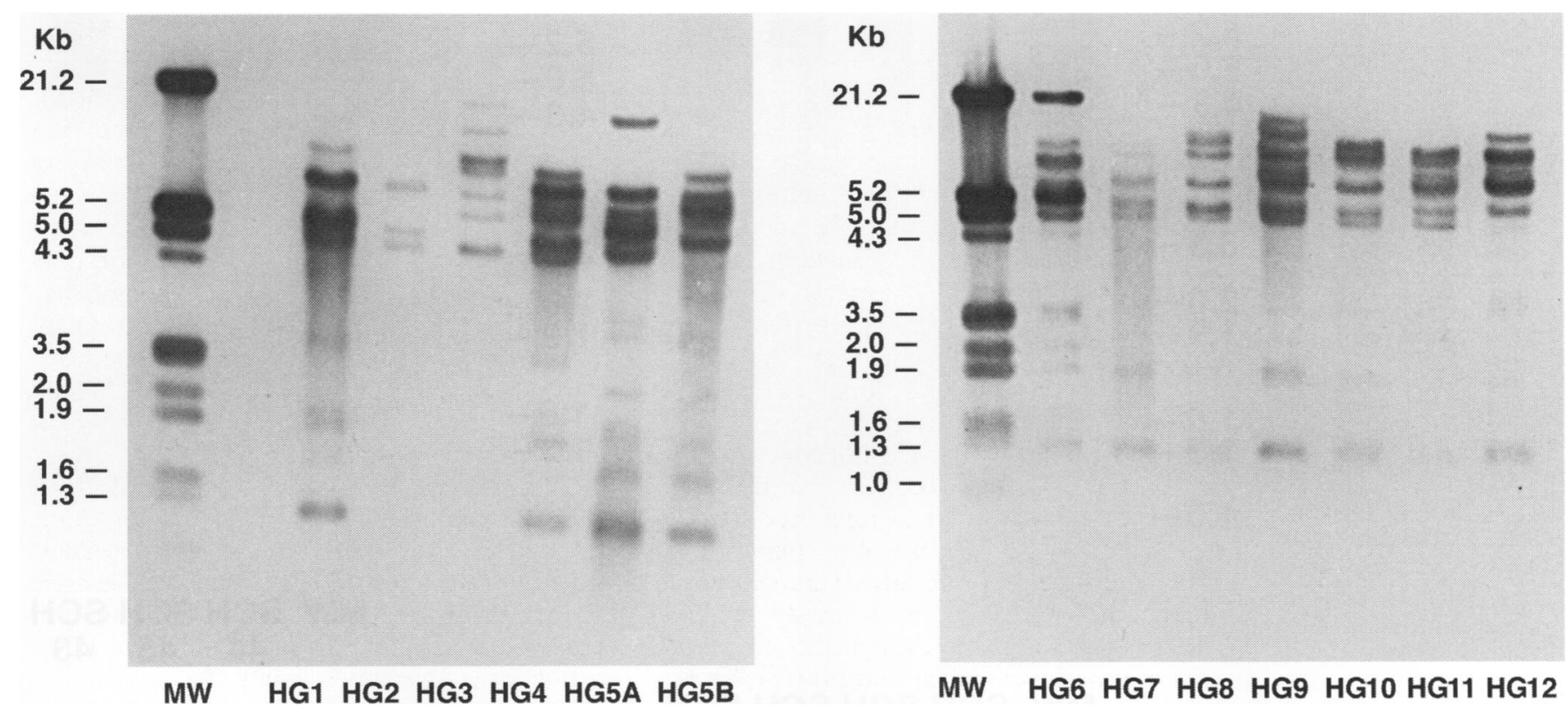

Figure 1 Ribotype patterns of reference strains of Aeromonas digested with Sma I (HG = Hybridisation group). 
Figure 2 Ribotype patterns of $A$ caviae strains digested with Sma I.

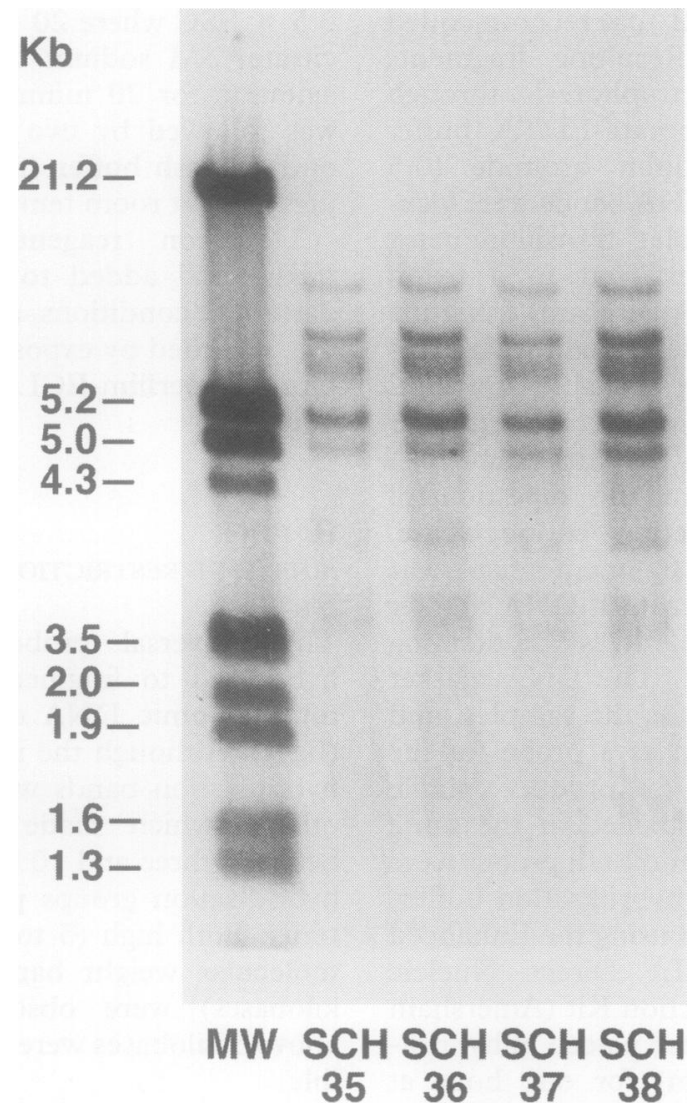

strains were indistinguishable and each pair of strains was also shown to have arisen from the same patient. Only two (SCH 44/45) of three strains of $A$ hydrophila (SCH 43/44/45) which had been isolated from the same patient and which were initially thought to be related

Figure 3 Ribotype patterns of $A$ caviae strains digested with Pst $I$.

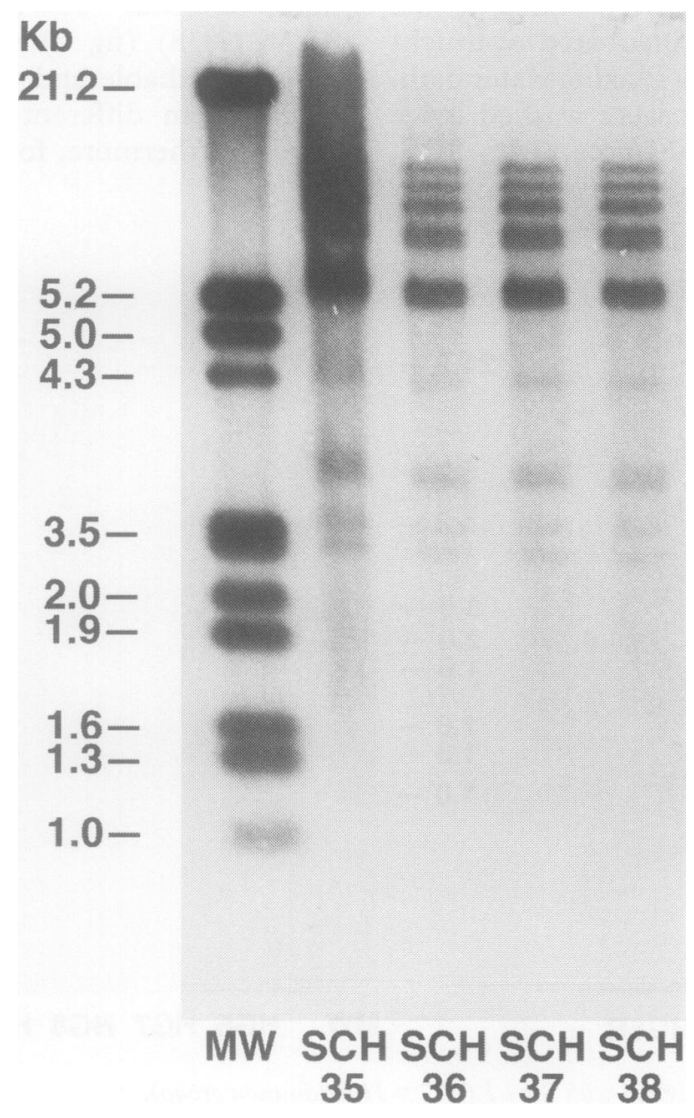

were also indistinguishable (results not shown).

rRNA GENE RESTRICTION PATTERNS USING Pst I Again, the universal probe hybridised to fragments of Pst I digested total genomic DNA from the reference strains (results not shown). Each reference strain had its own distinct pattern, and most of the observed bands were of a high molecular weight (5 to 21 kilobases). Although occasional low molecular weight bands were found, these were slightly larger in size than with Sma I and were in the range of 1.9 to 4.9 kilobases.

Similarly, the three phenospecies groups of clinical isolates all showed heterogeneous patterns and group allocation could not be made on ribotype pattern alone. However, as above, the same pairs of strains of both $A$ caviae and $A$ hydrophila were found to have originated from the same patients. The four $A$ caviae strains (SCH 35/36/37/38) (fig 3) were also indistinguishable with this restriction endonuclease. The same two (SCH 44/45) out of three strains of $A$ hydrophila (SCH 43/44/45) (fig 4) were also shown to be indistinguishable.

Reproducibility of the technique was confirmed by analysis of replicates prepared from separate bacterial colonies examined on different gels. Discrimination and typability of the technique were best achieved if light development of the chemiluminescent probe was restricted to one hour of exposure of autoradiographic film. This prevented the development of very strong bands which would have otherwise interfered with the visualisation of the weaker bands. At all times a molecular weight marker was used to ensure

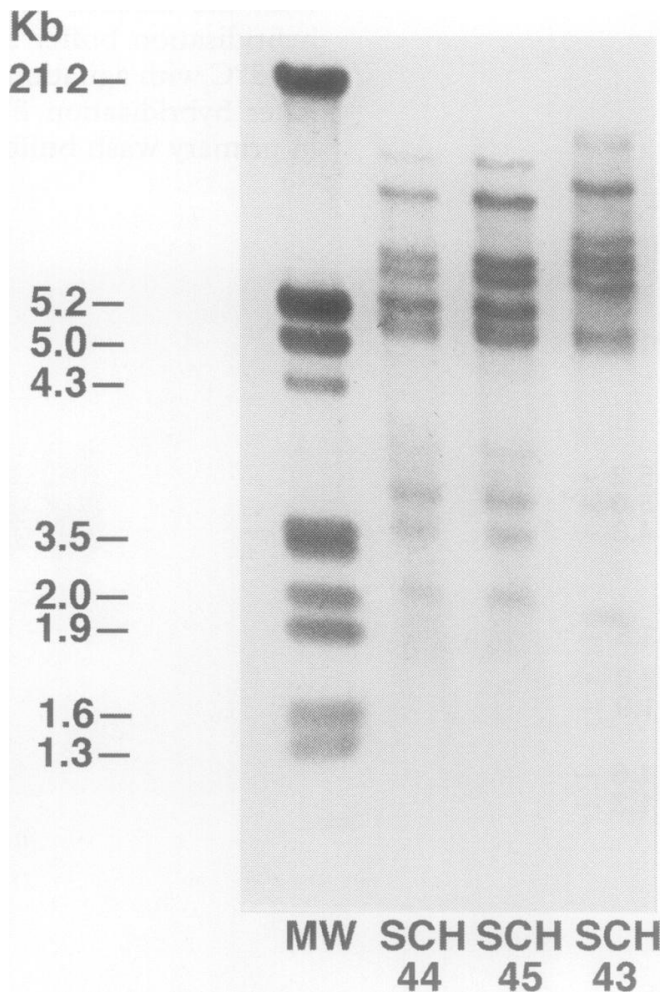

Figure 4 Ribotype patterns of $A$ hydrophila strains digested with Pst I. 
that the relative mobilities of the bands were consistent.

Ribotypes could not be obtained for a small number of strains (all $A$ sobria) due to incomplete digestion with either restriction endonuclease. However, out of a total of 15 restriction endonucleases that were tested, Sma I and Pst I produced the most useful ribotyping profiles after hybridisation. Overall, $25 \%$ of the clinical isolates failed to digest with Pst I and 13\% failed to digest with Sma I. After digestion with both restriction endonucleases (not in combination) extrapolated results showed that $90 \%$ of $A$ hydrophila strains produced a ribotype suitable for interpretation, $68 \%$ of $A$ caviae strains, and only $36 \%$ of $A$ sobria strains; $28 \%$ of $A$ sobria strains were typable using one of these enzymes and Sma I digested more of these strains than did Pst I. Moreover, because of the lack of association between phenospecies allocation and ribotype banding pattern, it was impossible to identify strains that in the previous study ${ }^{9}$ had shown discrepant results between biochemical findings and OMP analysis.

\section{Discussion}

Despite advances in taxonomical methodology it is still difficult for a diagnostic microbiology laboratory to identify strains of Aeromonas sp to the genospecies level. Moreover, even though laboratories are now more likely to identify isolates as $A$ hydrophila, $A$ sobria, or $A$ caviae, rather than just the $A$ hydrophila complex, problems still arise, due in part to a complicated taxonomy and the need to use many non-conventional biochemical tests. To relate disease symptoms to bacterial species, we need to be able to identify strains as accurately as possible. Several epidemiological surveys of Aeromonas sp have shown that of 11 hybridisation groups recovered from clinical material, three ( $A$ hydrophila-HG1, $A$ sobria-HG8, and $A$ caviae-HG4) account for more than $85 \%$ of all isolates. ${ }^{13-15}$ Our recent study, however, has shown that even with the use of several phenotypic methods, the identity of roughly $10 \%$ of strains remains uncertain. ${ }^{9}$ We therefore considered that despite the heterogeneity of aeromonads it would be worthwhile trying to apply a genotypic method such as ribotyping in an attempt to clarify this problem.

Unfortunately, two previous attempts to use ribotyping (with the combination of $16+$ $23 \mathrm{~S}$ rRNA) as a taxonomic tool for Aeromonas sp were unsuccessful. ${ }^{67}$ But these studies focused on the high molecular weight, intensively labelled bands. Compared with these previous studies we have been able to show stronger hybridisation with some of the lower molecular weight bands (which are now known to be the most useful, taxonomically) ${ }^{1}$ but there was still no discernible correlation between banding patterns and phenospecies. Moreover, it has recently been shown that a 567 base pair fragment of a 3 kilobase probe of the $16 \mathrm{~S}$ ribosomal gene is specific for hybridisation to low molecular weight DNA fragments, and gives a good correlation between rRNA patterns and hybridisation groups. This seems to be the best ribotyping method described to date for use as a taxonomical tool for Aeromonas sp, rather than using a universal probe $(16 S+23 S \text { rRNA })^{16}$ similar to the one used in this study. It is suggested, therefore, that as our and previously mentioned studies ${ }^{67}$ did not clearly demonstrate the lower molecular weight bands, despite using $16 \mathrm{~S}$ rRNA in a combined probe, this would have been unlikely to have been due to a dilution effect. Instead, the differences in the probe labelling method of $23 \mathrm{~S}$ rRNA were more likely to have had a direct effect on the labelling of 16S rRNA (reducing it).

It was evident from our study that a number of strains of both $A$ hydrophila and $A$ caviae produced indistinguishable ribotyping banding patterns. A retrospective analysis of clinical and microbiological findings showed that all paired strains were isolated from the same patient. However, this analysis helped elucidate some genotypic similarities in the following two examples.

Two specimens of faeces had been collected from each of two patients with gastroenteritis (SCH 35/36, SCH 37/38) admitted to the same ward within seven days of each other. Salmonella enteriditis and $A$ caviae were isolated from one patient, whereas only $A$ caviae was isolated from the second patient. Previously, all four strains of Aeromonas had been identified as $A$ caviae by conventional biochemical phenospeciation, and by use of the suicide phenomenon and aesculin hydrolysis, although they were unidentified by API 20 NE. ${ }^{8}$ OMP analysis performed on one strain only from each patient showed them to be similar and to be typical of the $A$ caviae group. ${ }^{9}$ Moreover, our ribotyping method, following digestion with either Sma I or Pst I, showed that all four strains were indistinguishable (figs 2 and 3 ) and that these strains could possibly relate to hospital cross-infection or could have arisen from the same point source.

In the second example three strains of $A$ hydrophila were isolated from the same patient (SCH 43/44/45) and were identical using biochemical tests (including the suicide phenomenon) and API $20 \mathrm{NE}$; unfortunately only one strain was tested by OMP analysis and this was typical of the $A$ hydrophila group. ${ }^{9}$ Using ribotyping, following digestion with either restriction endonuclease, two strains were shown to be indistinguishable but the third strain was dissimilar (fig 4). As with other strains of organisms that are similar phenotypically, a genotypic method is often required for differentiation and this raises questions as to the importance and prevalence of mixed infections caused by different strains of the same bacterial species. Our findings confirmed that ribotyping is useful for comparison of isolates of Aeromonas sp isolated from different patients and also for isolates from the same patient. 
The strength of this technology for epidemiological purposes has been realised for diverse Gram negative organisms ${ }^{17}$ and more recently for Aeromonas sp in particular. ${ }^{18} 19$ Indeed, it was this method that allowed the source of the first case of human intestinal Aeromonas infection to have its source identified (shrimp cocktail). ${ }^{19}$

In conclusion, we support the recent findings that ribotyping for epidemiological analysis of Aeromonas spp has great potential. ${ }^{18}$ Moreover, direct chemiluminescent labelling of $E$ coli rRNA produces a sensitive, universal probe suitable for clinical laboratory use in the investigation of outbreaks. Other advantages of this ribotyping labelling system are that all reagents are commercially available and the resulting labelled probe is, of course, nonisotopic. A disadvantage of this technique is that although most isolates of $A$ hydrophila and $A$ caviae could be ribotyped, about a third of the $A$ sobria strains failed to produce readable banding patterns. Our results indicated that this was primarily due to a lack of digestion with either Sma I or Pst I, which agrees with other findings, ${ }^{7}$ and that this was not caused by the extraction of insufficient DNA (data not shown). When we compared the relative activities of Sma I and Pst I against the three phenospecies, more isolates (25\%) could not be digested by the latter restriction endonuclease. Because of these findings, we suggest that ribotyping of Aeromonas sp should primarily follow DNA digestion with Sma I, and if any doubt is raised concerning the banding patterns produced, verification can be attempted using Pst I digestion.

Our findings indicate that the usefulness of this ribotyping method is limited by the relatively high proportion of untypable strains of A sobria. Interestingly, our previous taxonomical study of the same isolates also failed to characterise these strains by OMP analysis as there was a good deal of heterogeneity in the banding patterns. ${ }^{9}$ However, certainly for $A$ hydrophila and $A$ caviae, in comparison with other molecular typing methods such as plasmid analysis, OMPs, and isozyme profiles, ${ }^{672021}$ which have not been widely used for epidemiological investigations, ribotyping offers a major advantage. This is related to the fact that DNA analysis avoids the potential pitfalls associated with variable expression of phenotypes such as enzyme activities. It is more likely that larger diagnostic microbiology laboratories with suitable facilities would be able to use ribotyping as an epidemiological tool for Aeromonas sp as long as phenotypic complexities do not stand in the way of primary identification. If so, then ribotyping makes it possible to investigate the epidemiological links between isolates from patients and their environmental sources, which until now has been extremely difficult, even for reference laboratories, and has hindered our understanding of Aeromonas sp related diseases.

1 Martinetti-Lucchini G, Altwegg M. rRNA gene restriction patterns as taxonomic tools for the genus Aeromonas. Int f System Bacteriol 1992;42:384-9.

2 Carnahan AM, Behram S, Joseph SW. Aerokey II: a flexible key for identifying clinical Aeromonas species. 7 Clin Microbiol 1991;29:2843-9.

3 Abbott SL, Cheung WKW, Kroske-Bystrom S, Malekzadeh T, Janda JM. Identification of Aeromonas strains to the genospecies level in the clinical laboratory. strains to the genospecies level in

4 Woese CR. Bacterial evolution. Microbiol Rev 1987;51. 221-71.

5 Grimont F, Grimont PAD. Ribosomal ribonucleic acid gene restriction patterns as potential taxonomic tools. Ann Inst Pasteur Microbiol (Paris) 1986;137B:165-75.

6 Altwegg M, Altwegg-Bissig R, Demarta A, Peduzzi R Reeves MW, Swaminathan B. Comparison of four typing methods for Aeromonas species. $f$ Diarrhoeal Dis Res 1988;6:88-94.

7 Kuijper EJ, Van Elphen L, Leenders E, Zanen HC. Typing of Aeromonas strains by DNA restriction endonuclease analysis and polyacrylamide gel electrophoresis of cell envelopes. $尹$ Clin Microbiol 1989;27: trophoresis

8 Wilcox MH, Cook AM, Eley A, Spencer RC. Aeromonas spp as a potential cause of gastroenteritis in children. spp as a potential cause of $\mathrm{g}$.
$\mathcal{C}$ Clin Pathol $1992 ; 45: 959-63$.

9 Wilcox MH, Cook AM, Thickett KJ, Eley A, Spencer RC. Phenotypic methods for speciating clinical Aeromonas isolates. F Clin Pathol 1992;45:1079-83.

10 Akhtar N, Eley A. Restriction endonuclease analysis and ribotyping differentiate genital and non-genital strains of Bacteroides ureolyticus. $\mathcal{f}$ Clin Microbiol 1992;30: 2408-14.

11 Owen RJ, Borman P. A rapid biochemical method for purifying high molecular weight bacterial chromosomal DNA for restriction enzyme analysis. Nucleic Acids Res 1987;15:3631.

12 Maniatis T, Fritsch EF, Sambrook J. Molecular cloning: a laboratory manual. New York: Cold Spring Harbor Laboratory, 1982.

13 Altwegg M, Steigerwalt AG, Altwegg-Bissig R, LuthyOttenstein J, Brenner DJ. Biochemical identification of Aeromonas genospecies isolated from humans. $\mathcal{F}$ Clin Microbiol 1990;28:258-64.

14 Kokka RP, Janda JM, Oshiro LS, Altwegg M, Shimada T, Sakazaki $R$, et al. Biochemical and genetic characterisation of autoagglutinating phenotypes of Aeromonas associated with invasive and non-invasive disease. f Infect Dis 1991;163:890-4.

15 Kuijper EJ, Steigerwalt AG, Schoenmakers BSCIM, Peeters MF, Zanen HC, Brenner DJ. Phenotypic characterisation and DNA relatedness in human faecal isoacterisation and DNA relatedness in human faecal iso-

16 Gustaferro CA, Persing DH. Chemiluminescent universal probe for bacterial ribotyping. F Clin Microbiol 1992;30 1039-41.

17 Stull TL, LiPuma JJ, Edlind TD. A broad-spectrum probe for molecular epidemiology of bacteria: ribosomal RNA. f Infect Dis 1988;157:280-5.

18 Moyer NP, Martinetti G, Luthy-Hottenstein J, Altwegg M. Value of rRNA gene restriction patterns of Aeromonas spp for epidemiological investigations. Curr Microbiol 1992;24:15-21.

19 Altwegg M, Martinetti Lucchini G, Luthy-Hottenstein J, Rohrbach M. Aeromonas-associated gastroenteritis after consumption of contaminated shrimp. Eur $f$ Clin Microbiol Infect Dis 1991;10:44-5.

20 Chang BJ, Bolton SM. Plasmids and resistance to antimicrobial agents in Aeromonas sobria and Aeromonas hydrophila clinic

21 Picard B, Goullet P. Epidemiological complexity of hospital Aeromonas infections revealed by electrophoretic typing of esterases. Epidemiol Infect 1987;98:5-14. 